We report on a simple and safe method of transcutaneous gastrostomy (percutaneous endoscopic gastrostomy; PEG) replacement using the Seldinger technique in combination with vascular dilators. The procedure is illustrated in Figure $\mathbf{1}$ and $\mathbf{2}$.

We replaced 12 PEGs in eight patients over a period of 12 months. The median age of the patients was 36 years (range 23-88). The leading diagnosis in all patients was an underlying neurological disorder. In nine episodes the PEG had been accidentally removed. In three cases it had to be replaced because of malfunction, and the old PEG was removed using the "cut and push" technique [1]. Patients presented after a median delay of 17 hours (range 16-24). In ten cases the size of the replacement PEG was identical to or larger than that of the old one (14 Fr in eight patients and $10 \mathrm{Fr}$ in one patient; enlarged from $9 \mathrm{Fr}$ to $12 \mathrm{Fr}$ in one patient). In two cases the replacement PEG was smaller than the original one. In eight of the 12 patients the replacement was carried out as an outpatient procedure. The procedure was well tolerated, and no long-term complications were observed.

The Seldinger technique of PEG replacement was first reported in four cases of surgically created gastrostomies [2] but has not become widely accepted. Transcutaneous PEG replacement has also been described using Savary-Gilliard dilators (three cases) and Hegar's dilators (eight cases) $[3,4]$. This blind approach to dilation carries the potential risk of creating a false passage. The Seldinger technique has the advantage of preventing this by using the guide wire. This gives the replacement PEG more stiffness and makes replacement easy and atraumatic. Transcutaneous PEG replacement should be used with great care in patients with gastrostomies less than 4 weeks old. Fluoroscopy can confirm correct positioning of the wire under these circumstances.

\title{
A Simple and Safe Method of Transcutaneous Gastrostomy Replacement Using the Seldinger Technique
}

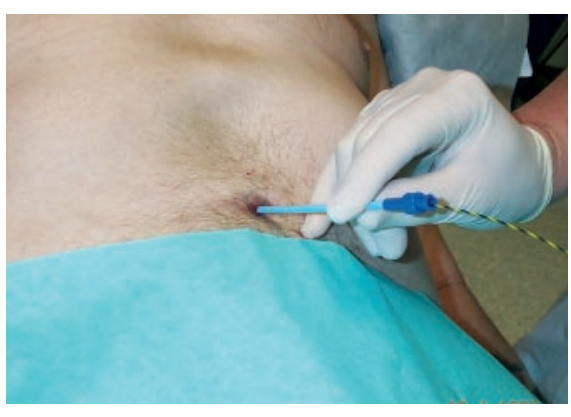

Figure 1 A standard endoscopic retrograde cholangiopancreatography (ERCP) guide wire (0.35-inch Jag-wire; Boston Scientific, Miami, USA) is passed through the fistulous tract until well into the stomach. Vascular dilators (Kimal, Uxbridge, England) are then passed over the guide wire to sequentially dilate the tract to $2 \mathrm{Fr}$ larger than the replacement tube, or until sufficient resistance or discomfort prevents further enlargement. The guide wire usually passes very easily into the stomach, but if resistance is met, withdrawal and further gentle probing usually locates the tract. Minor bleeding can occur which is selflimiting.

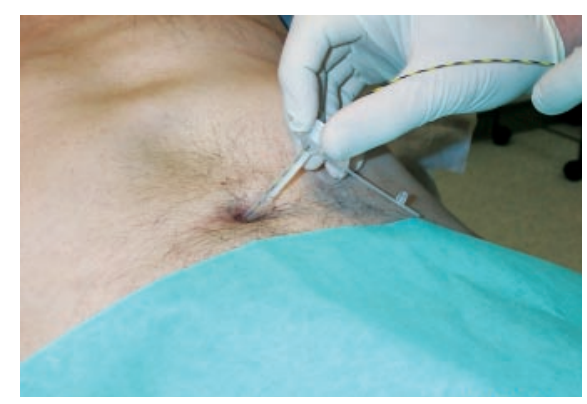

Figure 2 The gastrostomy tube is inserted through the fistula over the guide wire and the balloon inflated once in position. After aspiration of gastric contents to check for correct positioning, feeding can be started immediately.

\section{B. Frenz ${ }^{1}$, G. Siuda ${ }^{1}$, A. S. McIntyre ${ }^{2}$,}

S. P. L. Travis ${ }^{1}$

${ }^{1}$ Department of Gastroenterology, John

Radcliffe Hospital, Oxford, UK

2 Department of Gastroenterology,

Wycombe Hospital, High Wycombe, UK

\section{References}

${ }^{1}$ Pearce CB, Goggin PM, Collett J et al. The "cut and push" method of percutaneous endoscopic gastrostomy tube removal. Clin Nutr 2000; 19: 133-135

${ }^{2} \mathrm{Au}$ FC. Reinsertion of a gastrostomy tube using the Seldinger technique. JPEN J Parenter Enteral Nutr 1989; 13: 436-437

${ }^{3}$ Lopez Roses L, Gonzalez Ramirez A, Santos E et al. Delayed reposition of gastrostomy tube using Savary's dilators. Rev Esp Enferm Dig 1997; 89: 569-570 [In Spanish]

${ }^{4}$ Baker RC, Farnan TB, Gilroy D et al. Novel use of Hegar's dilators in gastrostomy tube reinsertion. Endoscopy 2000; 32: S13

\section{Corresponding Author}

\section{B. Frenz, M.D.}

Department of Gastroenterology John Radcliffe Hospital Headley Way, Headington Oxford, OX3 9DU United Kingdom Fax: +44-1865-220933 E-mail: mfrenz@doctors.org.uk 